Supplementary material

\title{
Kinetic modeling and optimization of sunflower oil methanolysis catalyzed by spherically-shaped $\mathrm{CaO} / \gamma-\mathrm{Al}_{2} \mathrm{O}_{3}$ catalyst
}

Dalibor M. Marinković, ${ }^{\text {la }}$ Marija R. Miladinović, ${ }^{2 b}$ Jelena M. Avramović, ${ }^{3 c}$ Miroslav V. Stanković, ${ }^{1 \mathrm{~d}}$ Olivera O. Stamenković, ${ }^{2 \mathrm{e}}$ Dušan M. Jovanović, ${ }^{\text {ff }}$ Vlada B. Veljković ${ }^{2 *}$

${ }^{1}$ University of Belgrade, Institute for Chemistry, Technology and Metallurgy (ICTM), Njegoševa 12, 11000 Belgrade, Serbia

${ }^{2}$ University of Niš, Faculty of Technology, Bulevar oslobodenja 124, 16000 Leskovac, Serbia

${ }^{3}$ Research and Development Center "ALFATEC" Ltd., Bul. Nikole Tesle 63/5, 18000 Niš, Serbia

The obtained full quadratic model equations with codded and actual factors are as follows, respectively:

$y=93.87-0.62 \cdot X_{1}+19.76 \cdot X_{2}+24.22 \cdot X_{3}-$

$-2.58 \cdot X_{1} \cdot X_{2}+6.84 \cdot X_{1} \cdot X_{3}-10.61 \cdot X_{2} \cdot X_{3}+$

$+0.0027 \cdot X_{1}^{2}-16.42 \cdot X_{2}^{2}-11.43 \cdot X_{3}^{2}$

and

$y=-211.37-3.76 \cdot X_{1}+693.56 \cdot X_{2}+40.27 \cdot 3-$

$-4.31 \cdot X_{1} \cdot X_{2}+1.14 \cdot X_{1} \cdot X_{3}-26.51 \cdot X_{2} \cdot X_{3}+$

$+0.0003 \cdot X_{1}^{2}-410.50 \cdot X_{2}^{2}-2.86 \cdot X_{3}^{2}$

\footnotetext{
* Corresponding author: Vlada B. Veljković, University of Niš, Faculty of Technology, 16000 Leskovac, Bulevar oslobođenja 124, Serbia; phone: +38116 247203; fax: +38116242859;

E-mail: veljkovicvb@yahoo.com.
} 
Table S1. CCCD matrix for the sunflower oil methanolysis over the $\mathrm{CaO} / \gamma-\mathrm{Al}_{2} \mathrm{O}_{3}$ catalyst.

\begin{tabular}{|c|c|c|c|c|c|c|c|c|c|}
\hline \multirow[t]{2}{*}{ No } & \multicolumn{3}{|c|}{ Coded factor } & \multicolumn{3}{|c|}{ Actual factor } & \multicolumn{2}{|c|}{ FAME content, $\%$} & \multirow{2}{*}{ RPD, \% } \\
\hline & $X_{1}$ & $x_{2}$ & $x_{3}$ & $X_{1}$ & $x_{2}$ & $x_{3}$ & Actual & Modeled $^{a}$ & \\
\hline 1 & -1 & -1 & -1 & 9 & 0.3 & 3 & 20.3 & 18.9 & +6.9 \\
\hline 2 & 1 & -1 & -1 & 15 & 0.3 & 3 & 4.1 & 4.0 & +2.4 \\
\hline 3 & -1 & 1 & -1 & 9 & 0.7 & 3 & 78.0 & 79.6 & -2.1 \\
\hline 4 & 1 & 1 & -1 & 15 & 0.7 & 3 & 59.0 & 64.7 & -9.7 \\
\hline 5 & -1 & -1 & 1 & 9 & 0.3 & 7 & 75.3 & 74.9 & +0.5 \\
\hline 6 & 1 & -1 & 1 & 15 & 0.3 & 7 & 94.0 & 87.3 & +7.1 \\
\hline 7 & -1 & 1 & 1 & 9 & 0.7 & 7 & 98.1 & 93.2 & +5.0 \\
\hline 8 & 1 & 1 & 1 & 15 & 0.7 & 7 & 98.9 & 105.6 & -6.8 \\
\hline 9 & -1.68 & 0 & 0 & 7 & 0.5 & 5 & 91.8 & 94.9 & -3.4 \\
\hline 10 & 1.68 & 0 & 0 & 17 & 0.5 & 5 & 96.1 & 92.8 & +3.4 \\
\hline 11 & 0 & -1.68 & 0 & 12 & 0.164 & 5 & 9.0 & 14.2 & -57.8 \\
\hline 12 & 0 & 1.68 & 0 & 12 & 0.836 & 5 & 86.0 & 80.7 & +6.2 \\
\hline 13 & 0 & 0 & -1.68 & 12 & 0.5 & 1.64 & 24.2 & 20.8 & +14.1 \\
\hline 14 & 0 & 0 & 1.68 & 12 & 0.5 & 8.36 & 99.0 & 102.3 & -3.3 \\
\hline 15 & 0 & 0 & 0 & 12 & 0.5 & 5 & 98.5 & 93.9 & +4.7 \\
\hline 16 & 0 & 0 & 0 & 12 & 0.5 & 5 & 96.2 & 93.9 & +2.4 \\
\hline 17 & 0 & 0 & 0 & 12 & 0.5 & 5 & 89.9 & 93.9 & -4.5 \\
\hline 18 & 0 & 0 & 0 & 12 & 0.5 & 5 & 98.1 & 93.9 & +4.3 \\
\hline 19 & 0 & 0 & 0 & 12 & 0.5 & 5 & 96.5 & 93.9 & +2.7 \\
\hline 20 & 0 & 0 & 0 & 12 & 0.5 & 5 & 84.0 & 93.9 & -11.8 \\
\hline & & & & & & & & MRPD, \% & \pm 2.0 \\
\hline
\end{tabular}

${ }^{\mathrm{a}} \mathrm{A}$ reduced regression model is used for calculation. RPD - relative percentage deviation, MRPD mean relative percentage deviation. 
Table S2 ANOVA analysis for the full quadratic model.

\begin{tabular}{l|rcrrrr}
\hline Source & $\begin{array}{r}\text { Sum of } \\
\text { squares }\end{array}$ & $\begin{array}{c}\text { Degrees of } \\
\text { freedom }\end{array}$ & $\begin{array}{c}\text { Mean } \\
\text { squere }\end{array}$ & F-value & $p$-value \\
\hline Model & 20052.06 & 9 & 2228.01 & 61.75 & $<0.0001$ \\
$X_{1}$ (Molar ratio) & 5.23 & 1 & 5.23 & 0.14 & 0.712 \\
$X_{2}$ (Catalyst loading) & 5332.37 & 1 & 5332.37 & 147.79 & $<0.0001$ \\
$X_{3}$ (Reaction time) & 8009.88 & 1 & 8009.88 & 222.01 & $<0.0001$ \\
$X_{1} X_{2}$ & 53.46 & 1 & 53.46 & 1.48 & 0.252 \\
$X_{1} X_{3}$ & 374.28 & 1 & 374.28 & 10.37 & 0.0092 \\
$X_{2} X_{3}$ & 899.73 & 1 & 899.73 & 24.94 & 0.0005 \\
$X_{1}^{2}$ & $1.052 \cdot \mathrm{e}^{-4}$ & 1 & $1.052 \cdot \mathrm{e}^{-4}$ & $6.00 \cdot \mathrm{e}^{-6}$ & 0.999 \\
$X_{2}^{2}$ & 3885.45 & 1 & 3885.45 & 107.69 & $<0.0001$ \\
$X_{3}^{2}$ & 1883.74 & 1 & 1883.74 & 52.21 & $<0.0001$ \\
\hline Pure error & 164.85 & 5 & 32.97 & & \\
Lack-of-fit & 195.94 & 5 & 39.19 & 1.19 & 0.427 \\
Total correction & 20412.85 & 19 & & & \\
\hline$R^{2}=0.982 ; R_{a d j}^{2}=0.966 ; R_{p r e d}^{2}=0.913 ;$ C.V. $=8.0 \%$ and Adequate Precision = 22.7
\end{tabular}



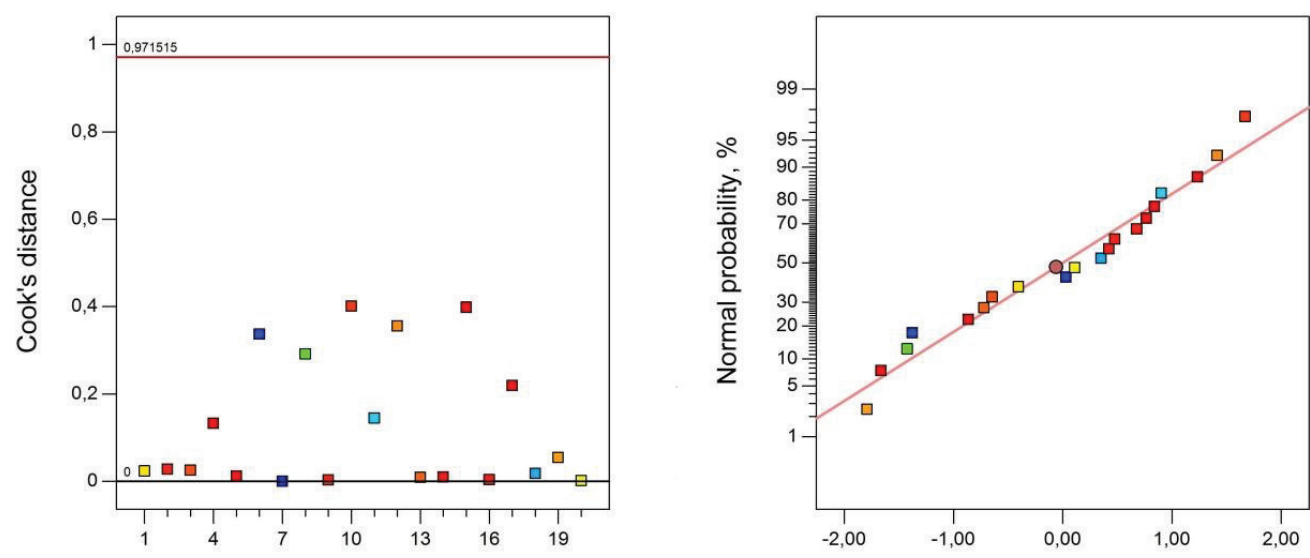

Run number

a)

Internally studentized residuals

b)
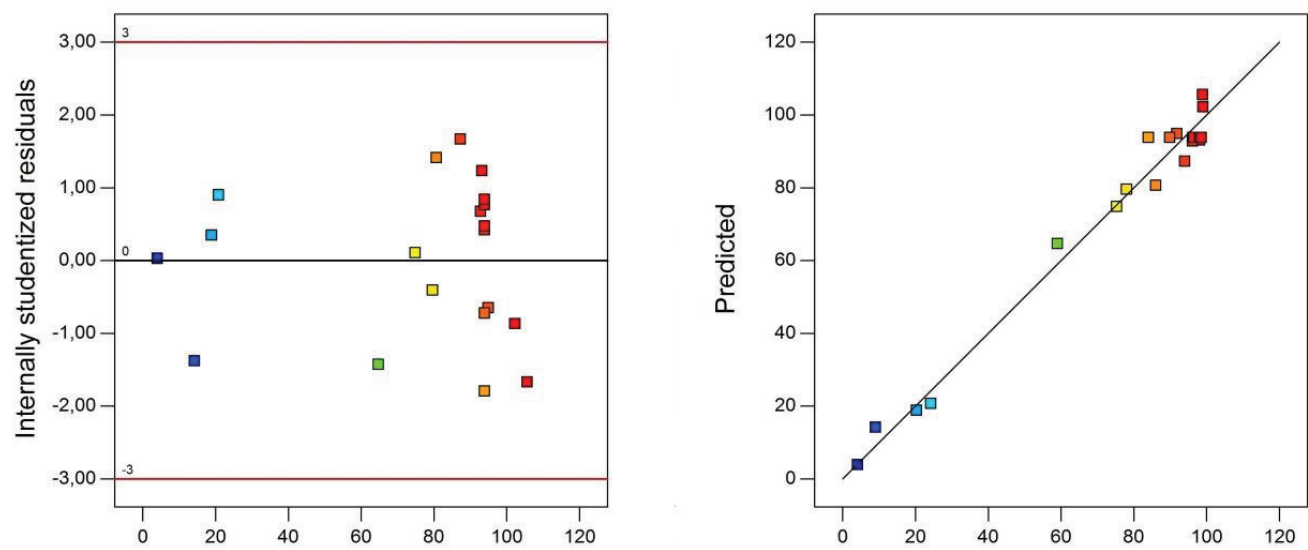

Predicted

c)

Actual

d)

Figure S1 Statistical evaluations of the reduced quadratic model: (a) Cook's distance, (b) normal probability, (c) model outliers and (d) actual and predicted FAME content plot. 